\author{
ABSTRACT \\ Space Vehicle Pose Estimation via Optical Correlation and Nonlinear Estimation \\ John Rakoczy/MSFC \\ Kenneth Herren/MSFC
}

A technique for 6-degree-of-freedom (6DOF) pose estimation of space vehicles is being developed. This technique draws upon recent developments in implementing optical correlation measurements in a nonlinear estimator, which relates the optical correlation measurements to the pose states (orientation and position). For the optical correlator, the use of both conjugate filters and binary, phase-only filters in the design of synthetic discriminant function (SDF) filters is explored. A static neural network is trained a priori and used as the nonlinear estimator. New commercial animation and image rendering software is exploited to design the SDF filters and to generate a large filter set with which to train the neural network. The technique is applied to pose estimation for rendezvous and docking of free-flying spacecraft and to terrestrial surface mobility systems for NASA's Vision for Space Exploration. Quantitative pose estimation performance will be reported. Advantages and disadvantages of the implementation of this technique are discussed. 


\title{
Space vehicle pose estimation via optical correlation and nonlinear estimation
}

\author{
John M. Rakoczy \& Kenneth A. Herren \\ NASA Marshall Space Flight Center \\ Huntsville, Alabama
}

\begin{abstract}
A technique for 6-degree-of-freedom (6DOF) pose estimation of space vehicles is being developed. This technique draws upon recent developments in implementing optical correlation measurements in a nonlinear estimator, which relates the optical correlation measurements to the pose states (orientation and position). For the optical correlator, the use of both conjugate filters and binary, phase-only filters in the design of synthetic discriminant function (SDF) filters is explored. A static neural network is trained a priori and used as the nonlinear estimator. New commercial animation and image rendering software is exploited to design the SDF filters and to generate a large filter set with which to train the neural network. The technique is applied to pose estimation for rendezvous and docking of free-flying spacecraft and to terrestrial surface mobility systems for NASA's Vision for Space Exploration. Quantitative pose estimation performance will be reported. Advantages and disadvantages of the implementation of this technique are discussed.
\end{abstract}

Keywords: Pose estimation, optical correlation, neural networks, rendezvous \& docking, binary phase-only filters

\section{Introduction}

This paper presents an effort to apply optical correlation to pose estimation sensors for rendezvous and docking of freeflying spacecraft and space-based surface mobility systems. Two significant advances in recent years have motivated this current attempt at applying optical correlators to space-based pose estimation. The first advance is the development of high-speed optical correlators. An optical correlator has been recently developed which can perform up to 4000 correlations per second ${ }^{1}$. This correlator is capable of comparing a single spacecraft image to a very large database of images in a reasonable amount of time. When using synthetic discriminant function (SDF) filters, this correlator could identify an object from a database of thousands of images or poses within a single second. Such a correlator has the potential of enabling a sensor that could yield pose estimates on the order of 1-10 Hertz, a reasonable bandwidth for space applications. The second advance is the emergence of sophisticated solid modeling and animation software running on desktop computers with ever growing processing speed and memory allocations. These new software tools, such as 3ds Max by Autodesk, facilitate the generation of a multitude of images for use as matched filters in an optical correlator. The animation software enables simulation of objects with realistic lighting conditions and camera parameters. Many SDF filters could be designed, a priori, relatively rapidly using computer-generated filter databases. These two advances, when incorporated with linear and nonlinear estimation techniques (including artificial neural networks) give merit to new efforts to use optical correlation techniques for space vehicle pose estimation ${ }^{2}$.

Castro $^{3,4}$ proposed an approach to pose estimation that shows promise. Figure 1 graphically illustrates their technique. Within this framework, the high-speed optical correlator would be used to evaluate the correlations and provide the measurements to the pose estimator. The pose estimator, residing on the spacecraft on-board computer, estimates the vehicle states or poses (attitude and position) as a function of the measurements. The pose estimator is the inverse of a transformation from a space comprising the poses to a space comprising the correlation measurements. The transformation is, in general form, nonlinear so a nonlinear estimator would be a natural solution. Artificial neural networks are well suited to nonlinear estimation problems and were investigated by Castro for this application. In certain smaller regimes, a linear estimator may yield good estimates and be a good approximation to the nonlinear estimator as also shown by Castro. 


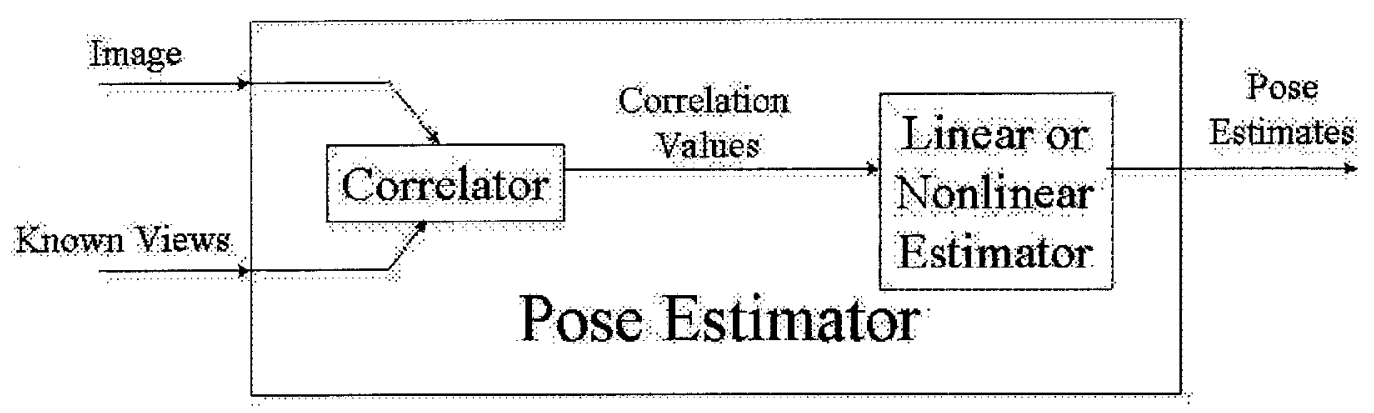

Figure 1: Pose Estimator Concept

In our current attempt at space vehicle pose estimation, we adopted the Castro technique, extended it from 2 degrees-offreedom (DOF) to 3DOF, and looked at the practical implementation using binary phase-only filters (BPOF) on the Boulder Nonlinear Systems correlator ${ }^{1}$. Castro estimated a recognition flag as one of the states in the pose estimator of Figure 1. That approach often resulted in false detections of images not within the detection class, especially with linear correlations. We propose to remedy that ambiguity with a 2-step approach. The first step is an interclass discrimination step, in which the class of the test image would be ascertained. The second step is the pose estimation step, implementing the concept in Figure 1.

In this paper we report on the theoretical feasibility of this pose estimation technique, present quantitative performance results, and discuss issues associated with its implementation in real hardware. Section 2 gives a general description of the 2-step process: class distinction and pose estimation. Section 3 describes the implementation of the 2 -step process using conjugate matched filters with both linear and nonlinear estimators. Section 4 describes the adaptation of the 2step process for realization in a high-speed optical correlator using binary phase-only synthetic discriminant function (BPOSDF) filters for the detection step and binary phase-only filters (BPOF) for the pose estimation step. Section 5 discusses some of the practical considerations in implementing this type of pose estimator. Section 5 also presents some general observations and some lessons learned from the development of this pose estimator.

\section{Description of 2-step process}

We propose a pose estimation methodology involving a 2-step process. The first step is an interclass discrimination step. The interclass discrimination step processes the observed image and determines which "class" or subset of poses to which it belongs. We intend to perform the interclass distinction using synthetic discriminant function (SDF) filters in a manner first suggested by Casasent ${ }^{5}$. The second step is a linear pose estimation step within the class identified in step one. The second step involves a relatively small bank of "construction" filters within the discerned class. This bank of filters is used to compute, a priori, a linear mapping between the poses represented by the construction filters and the auto- and cross-correlations of the construction filters with themselves. It is well known that the relationship between pose and correlation is not nicely linear, but under certain conditions, a linear approximation might be sufficient for some kinds of pose estimation requirements. The remainder of this section describes the mathematical operations involved in each step of the 2-step pose estimation process.

A classical SDF is defined by equation 1:

$$
H_{k}=\sum_{i=1}^{N} a_{i} F_{i}
$$


The $k$ th SDF is a weighted superposition of a set of $\mathrm{N}$ filters, $F$. The filters, $F$, are the complex conjugates of the Fourier transforms of the images in the construction set. The $a$ are the weight coefficients on each filter. The weight coefficients are computed by equation 2 :

$$
a=R^{-1} C
$$

$C$ is a Nx1 vector comprising prescribed correlation values for the resultant SDF. The matrix, $R$, is the NxN matrix of correlations among the $\mathrm{N}$ images in the construction set. For conjugate matched filters, $R$ is symmetric and thereby, full rank and invertible. The selection of the vector $C$ becomes the key in designing the SDF. In this paper we investigated two approaches to selecting $C$, the multilevel nonredundant filter method and the equal correlation peak method ${ }^{5}$. Each of these two approaches has its own merits and drawbacks, and some of them will be exposed in our application to conjugate filters and binary phase-only filters.

The second step is the pose estimation step. For this solution we borrow a technique from Castro. We seek to establish a relationship or mapping from the object poses to the numerical values obtained by correlating images of known poses with the observed 2-dimensional image. Equation 3 is an equation that might govern such a relationship.

$$
C=T P
$$

The $\mathrm{Nx} 1$ vector $C$ comprises the $\mathrm{N}$ correlations that were evaluated using the $\mathrm{N}$ construction filters within the class of interest. The vector $P$ is the $3 \times 1$ vector comprising the 3 pose degrees-of-freedom (DOF). The matrix $T$ is a $\mathrm{Nx} 3$ matrix which is a linear mapping from poses to correlations for that particular set of construction filters. If one has $\mathrm{N}$ construction filters representing $\mathrm{N}$ distinct poses, then $P$ would become $3 \mathrm{xN}$, and the $C$-matrix would become $\mathrm{NxN}$, the correlation matrix of the construction filters with themselves.

The linear estimator is obtained by computing the pseudo-inverse of $T$. The preferred method for computing the linear estimator is shown in equation 4.

$$
T^{+}=P C^{-1}
$$

Since $C=R$, the correlation matrix is full-rank and invertible. Computation of the pseudo-inverse of $T$ by equation 4 minimizes estimator errors arising from poor numerical conditions. Otherwise, one could solve for $T$ by taking the pseudo-inverse of $P$ and premultiplying it to $C$. Then one would explicitly evaluate the pseudo-inverse of $T$ by conventional methods. This latter approach introduces significant numerical errors first, in the pseudo-inverse of $P$, which is highly rectangular, and second in the subsequent pseudo-inverse of an already poorly computed matrix comprised of the pseudo-inverse of $P$.

The poses can be estimated by equation 5 .

$$
\hat{P}=T^{+} C_{\text {meas }}
$$

The vector $C_{\text {meas }}$ is a vector comprising the $\mathrm{N}$ measured correlations of the observed image with the $\mathrm{N}$ construction filters in the filter bank of this particular class. Equation 5 yields an estimate of the poses based on a linear approximation to the mapping of poses to correlations even though the relationship, for both conjugate and BPOF filters, is nonlinear. Equation 6 expresses this relationship in general form.

$$
C=T(P)
$$

Equation 6 shows $T$ as a general, nonlinear function of the poses $P$. Then a nonlinear pose estimator would look like equation 7. 


$$
\hat{P}=T^{\prime}(C)
$$

In equation $7 T^{\prime}$ is the nonlinear estimator, which is a nonlinear function of the correlations $C$. Below we design the nonlinear-estimator by training, a priori, an artificial neural network.

\section{Discussion of 2-step process using conjugate matched filters}

This section describes the 2-step pose estimation process using exclusively conjugate matched filters. The first step is the interclass discrimination step. In the interclass discrimination step a SDF is designed in order to determine the class to which the observed image belongs. The SDF was designed using the multilevel nonredundant filter approach described by Casasent. We next describe how we applied that approach to the example problem of Space Shuttle pose estimation.

Figures 2a-d depict four different "classes" of Space Shuttle poses. In other applications "class" may mean an entirely different object. Here "class" means a distinctly different Space Shuttle orientation or pose. The images were obtained from a Space Shuttle model in the 3ds Max animation software from Autodesk. Each of the four classes was assigned a prescribed correlation value. The prescribed correlation values comprise the vector $C=[150,000120,00090,000$ $60,000]^{\mathrm{T}}$. The prescribed correlation values were chosen to establish a significant threshold between the different classes. In this way a single SDF is intended to distinguish 4 different classes using 4 different levels of correlation values. Thus it is a nonredundant multilevel filter.

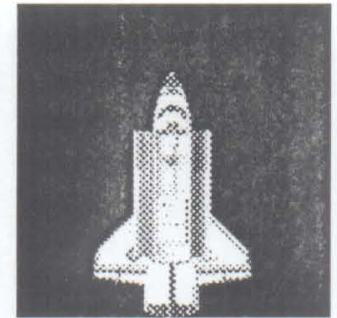

Figure 2a: Class 1

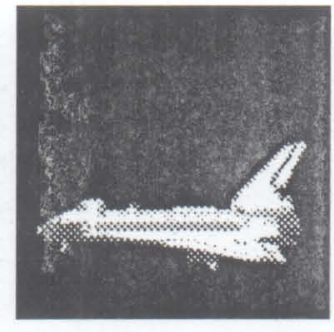

Figure 2b: Class 2

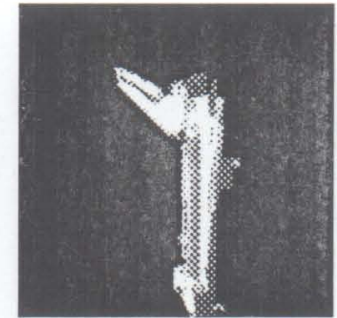

Figure 2c: Class 3

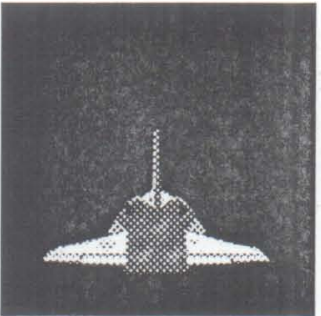

Figure 2d: Class 4

A single SDF, $H$, was designed according to equations 1 and 2 and implemented in equation 8:

$$
H=\sum_{i=1}^{4} a_{i} F_{i}
$$

The $F$ are the complex conjugates of the Fourier transforms of each image in Figure 2. The $a$ are the weighting coefficients on each of the filters representing the four images. The weighting coefficients, $a$, are computed from equation 2. In this case the matrix, $R$, is the $4 \times 4$ matrix of correlations among the four images in Figure 2 . For this particular set of images and the prescribed correlations in $C$, the solution becomes $a=\left[\begin{array}{ll}7.17 & 15.4712 .70 \\ 8.93\end{array}\right]^{\mathrm{T}}$.

The SDF was synthesized by substituting the vector $a$ into equation 8 . Next, the SDF was tested on a set of images. The test image set was obtained from 3ds Max by generating 125 images from 0-8 degrees in pitch, yaw and roll about the orientation in Figure 2a. The images were generated in 2-degree increments. Correlations were then computed for each test image with the SDF, $H$. The correlation values are plotted in Figure 3. Note that all the correlation values are above 132,000 . That magnitude clearly exceeds the threshold of 120,000 for the second class of Space Shuttle poses from Figure 2. This test indicates that this particular SDF is a good interclass discriminator for poses within 8 degrees of Figure 2a in pitch, yaw and roll. 


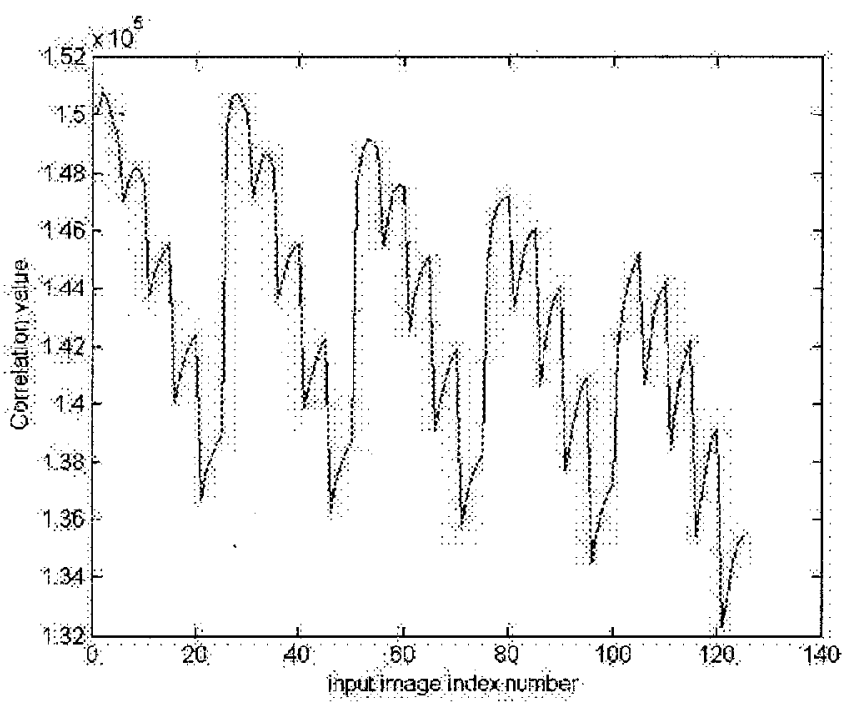

Figure 3: Correlations via SDF discriminator
After the images were determined to be within the class of Figure $2 \mathrm{a}$, the linear estimator specifically designed for class 1 was used to estimate the pose of each of the test images. The linear estimator was designed using 27 construction filters. The 27 filters were the complex conjugates of the Fourier transforms of 27 of the test images. The 27 construction filters were chosen to correspond to the corners, faces, edges and center of an $8 \times 8 \times 8$ degree cube, which spanned the space of class 1 . Construction filters were at the center of the cube and at each of the eight corners. A construction filter was selected at the center of each of the six faces, and at the midpoint of each of the 12 edges of the cube. Equation 4 was used to compute the $3 \times 27$ estimator matrix relating correlations to poses. The resultant estimator matrix interpolated the poses within the $8 \times 8 \times 8$ cube, given the measured correlation value.

The performance of the linear estimator was evaluated using 189 test images within the class- 1 cube. Each of the 125 images was correlated with the 27 construction filters. The values were stored in 189 separate $27 \times 1$ vectors. The $3 \times 27$ linear estimator was multiplied to each correlation vector. After running all 189 test images through the linear estimator, statistics were compiled for the estimator's accuracy within this class. Pitch (out-of-plane) accuracy was 0.22 degrees RMS. Yaw'(in-plane) accuracy was 0.20 degrees RMS. Roll (out-of-plane) accuracy was 0.14 degrees RMS. These results are comparable to those reported previously for a $2 \mathrm{DOF}$ linear estimator ${ }^{3,4}$.

Next an artificial neural network (ANN) was designed in order to attempt to improve the accuracy of the linear estimator. Castro reported a significant gain in performance with ANNs on the 2DOF problem. We attempted to apply an ANN to the 3DOF problem. An estimator in the form of equation 7 was implemented in a 2-layer back-propagation network trained by the Levenberg-Marquardt algorithm. The ANN used 27 correlations as the input, just like the linear

- estimator. Best results were obtained with 60 neurons in the hidden layer and 40 training epochs. 125 of the 189 test images were used as "training" filters for the neural network. Estimator performance was assessed by evaluating all 189 test images. Pitch accuracy was 0.22 degrees RMS. Yaw accuracy was 0.17 degrees RMS, and roll accuracy was 0.14 degrees RMS.

The results from the ANN show slight improvement over the linear estimator, but not very much. Since the ANN had a very high accuracy on the 125 images in the training set, the statistics were skewed by the training poses. We were interested in how well the ANN estimated those poses that were not in the training set. Statistics for the 64 non-training filters were significantly worse. Pitch accuracy was 0.40 degrees RMS. Yaw accuracy was 0.29 degrees RMS, and roll accuracy was 0.24 degrees RMS. These results indicate that the ANN was over-fittiing between the points in the training set.

\section{2-step process using BPOSDF and BPOF}

Since the ultimate goal of this undertaking was to implement the pose estimator in a real high-speed optical correlator using spatial light modulators (SLM), exclusively real-valued filters are required. Binary phase only filters (BPOF) are well suited for this application since their values are either 1 or -1 . In our 2-step pose estimation process, the first challenge in implementation is to convert the conjugate matched SDF into a BPOSDF for the class-distinction step. It is expected that the BPOSDF, being a discrete approximation to the conjugate matched filter, will not behave exactly as 
the conjugate SDF did, and some accommodations might have to be made. In the second step, the linear estimation step, the construction set of filters must be converted to BPOFs. The linear estimator must be redesigned to accommodate the correlations with the BPOFs, and degradations in performance are expected here for the same reasons as expected for the BPOSDF.

The computation of the BPOSDF poses some difficulties that do not arise with simple conjugate matched filters. Whereas the conjugate matched SDF uses equations 1 and 2 to solve for the SDF weight coefficients exactly, the BPOSDF cannot be computed that way. If the $F$ were phase-only binarized prior to evaluating the correlation matrix $R$ and substituting into equation 2 , the resultant SDF, $H$, is anything but binary and phase only. Then $H$ would have to be phase-only binarized, but the correlation matrix would no longer be valid because the correlations with $H$ would be significantly different than those in the correlation matrix for which the weighting coefficients were designed. The design of the BPOSDF is a nonlinear optimization problem which can be solved iteratively ${ }^{6,7}$. We chose to compute the BPOSDF by use of a genetic algorithm because genetic algorithms have proven successful in applications like this ${ }^{8}$. The genetic algorithm toolbox from MATLAB facilitates this solution technique. Figure 4 is a flow diagram illustrating the process used in computing the BPOSDF.

The first step is to start with the four conjugate matched filters of the images in Figure 1, equation 1 and an initial population of guesses on the $a$ vectors. Next we compute $H$ for every $a$ in the population. We chose a population of 50 so there will be $50 \mathrm{H}$. Next, each $H$ is phase-only binarized. Then $504 \times 1$ correlation vectors are computed by correlating each $H$ with the four images from Figure 1. Next we compute the mean-square error between each of the 50 $4 \mathrm{x} 1$ correlation vectors and the prescribed correlation vector, $C_{p r e}$. The mean square error difference from the previous iteration is evaluated as the algorithm stopping criteria. If the stopping criteria is not met, the genetic algorithm operates on the population of $a$ by selection, crossover and mutation to obtain a new population of $a$ for the next iteration. When the stopping criteria is finally met (in about 20-30 iterations), the $a$ which provide the best mean square error are chosen as the weighting coefficients. Once the weighting coefficients are determined, equation 1 is evaluated, and $H$ is phaseonly binarized to compute the BPOSDF.

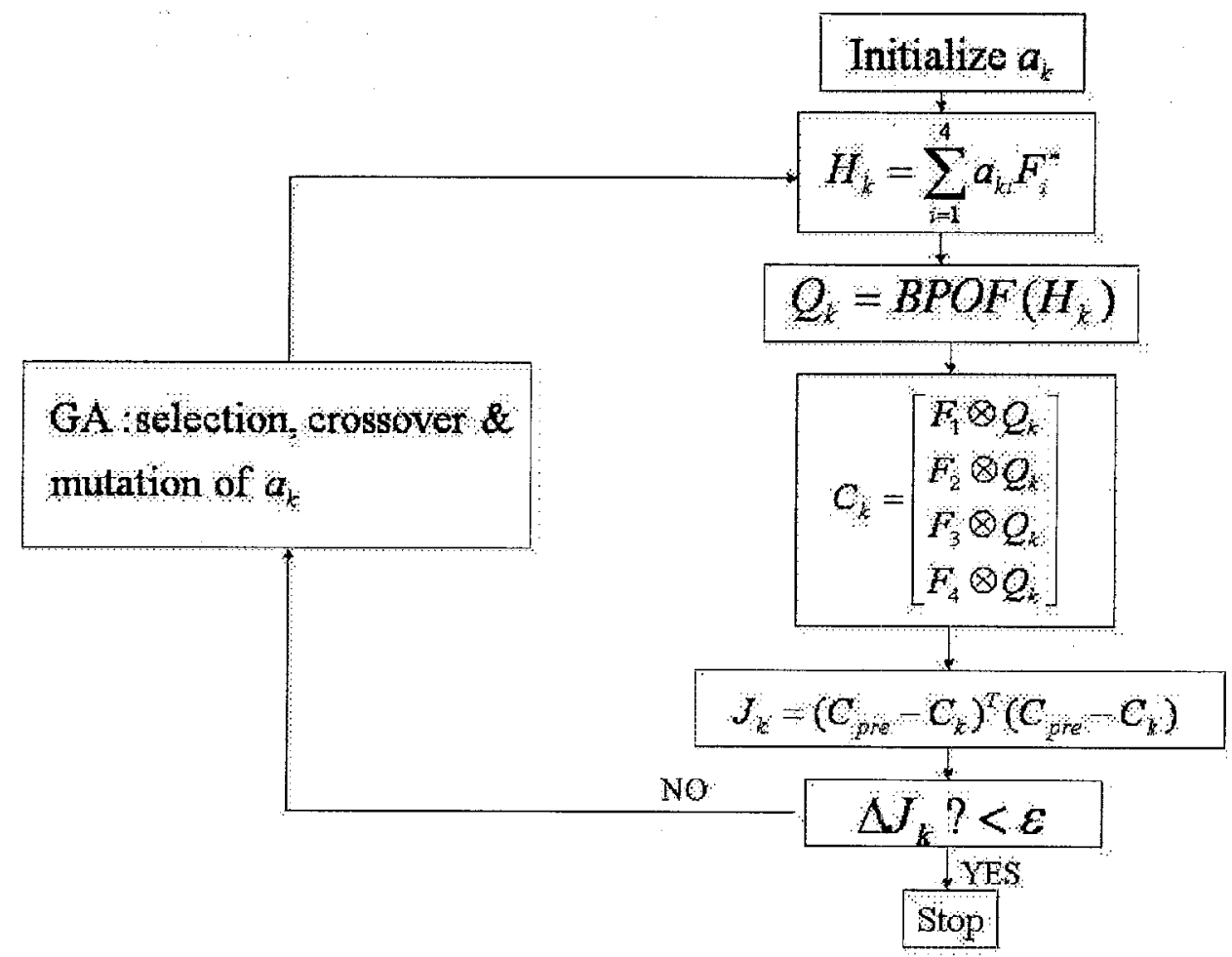

Figure 4: Genetic Algorithm to design BPOSDF 
We again chose to use a single multilevel nonredundant SDF. For the BPOSDF design, the prescribed correlation vector was $C=\left[\begin{array}{llll}2500 & 2000 & 1500 & 1000\end{array}\right]^{\mathrm{T}}$. The correlation values obtained from the resultant BPOSDF were $C=[1800$ $13301040600]^{\mathrm{T}}$. The discrepancy is attributable to the fact that, unlike the conjugate SDF, one cannot exactly satisfy the prescribed conditions with a BPOSDF. There is no guarantee of an exact solution with the BPOSDF because of the phase-only and binarizing operations. Nevertheless, the algorithm converges to a best-fit solution that yields significant thresholds between the individual classes.

Next, the BPOSDF was applied to 125 test images. Figure 5 shows the correlation values. Note that most of the correlations are below the threshold for class 2 , and many of the values are below the thresholds for class 3 and 4 . This indicates that this multilevel BPOSDF is not a good filter for class discrimination for these classes. This is attributable to BPOFs being such good discriminators. The BPOFs are very good at intra-class discrimination, which makes them very bad for inter-class discrimination on this particular set of images. As a consequence of this result, it was necessary to discard the nonredundant multilevel BPOSDF and design a different type of BPOSDF to perform the inter-class discrimination.

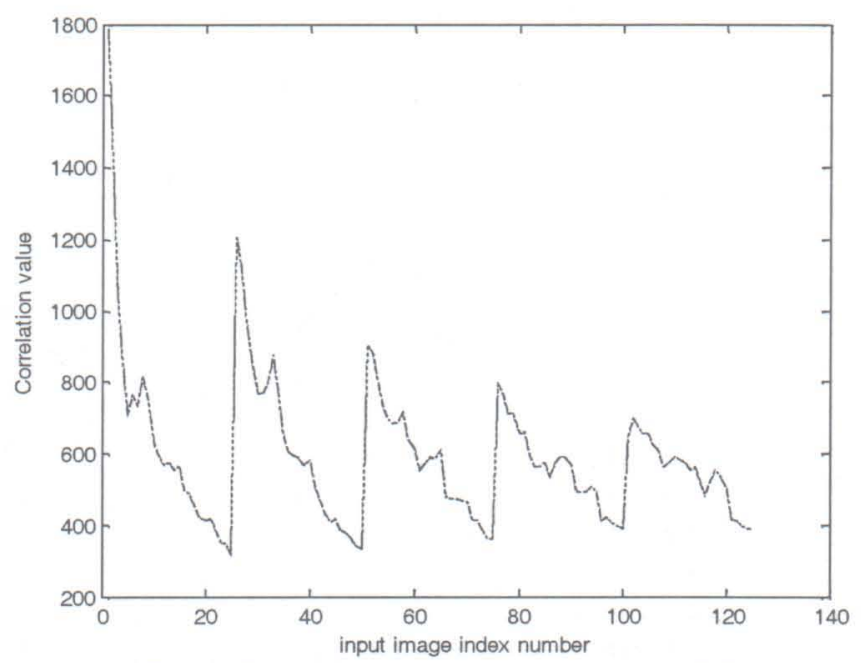

Figure 5: Correlations via multilevel BPOSDF

A new BPOSDF inter-class discriminator was designed using filters within class 1 . The filter design approach was the equal correlation peak (ECP) method ${ }^{5}$. The three images shown in Figure 6a-c were used in the BPOSDF design. Figure $6 \mathrm{a}$ is at $(0,0,0)$ degrees (pitch, yaw, roll). Figure $6 \mathrm{~b}$ is at $(4,4,4)$ degrees, and Figure $6 \mathrm{c}$ is at $(8,8,8)$ degrees. The prescribed correlation vector contained equal magnitudes for each of the 3 filters in the construction set. The resultant correlation values with the computed BPOSDF were $C=\left[\begin{array}{llll}1772 & 1777 & 1741\end{array}\right]^{\mathrm{T}}$. This BPOSDF was designed with the expectation that the correlation values for the other images within the $(0,0,0)-(8,8,8)$ class would be above the noise level and above the level of this BPOSDF's correlation with the images from classes 2, 3 and 4.

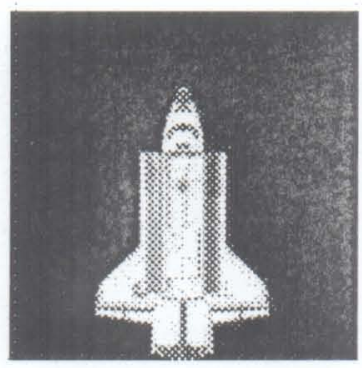

Figure 6a: $(\mathrm{P}, \mathrm{Y}, \mathrm{R})=(0,0,0)$

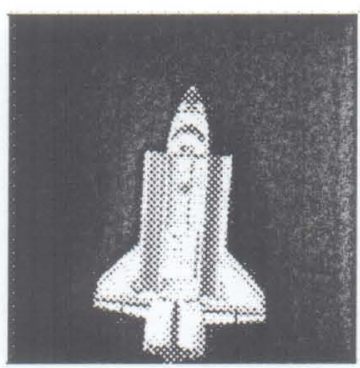

Figure 6b: $(\mathrm{P}, \mathrm{Y}, \mathrm{R})=(4,4,4)$

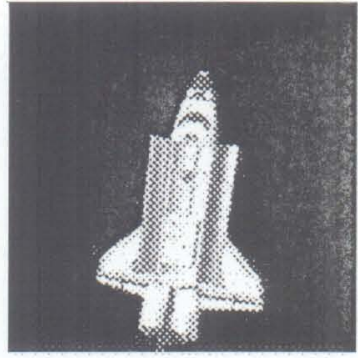

Figure 6c: $(\mathrm{P}, \mathrm{Y}, \mathrm{R})=(8,8,8)$

The new ECP BPOSDF was used to compute correlations with 125 images within class 1 . Figure 7 shows the resulting correlation values. All correlations within class 1 were above 643. The three peaks are the correlations for the 3 construction images from Figure 6. The noise floor for all 125 correlations was around 250 so there was sufficient signal-to-noise ratio to discern a true correlation. Table 1 shows a comparison of class 1 correlations against correlations with class 2, 3 and 4 images. All images from class 2, 3 and 4 had correlation values below 221, putting 
them under the noise floor for this BPOSDF. The results indicate that this ECP BPOSDF is a sufficient filter for interclass discrimination.

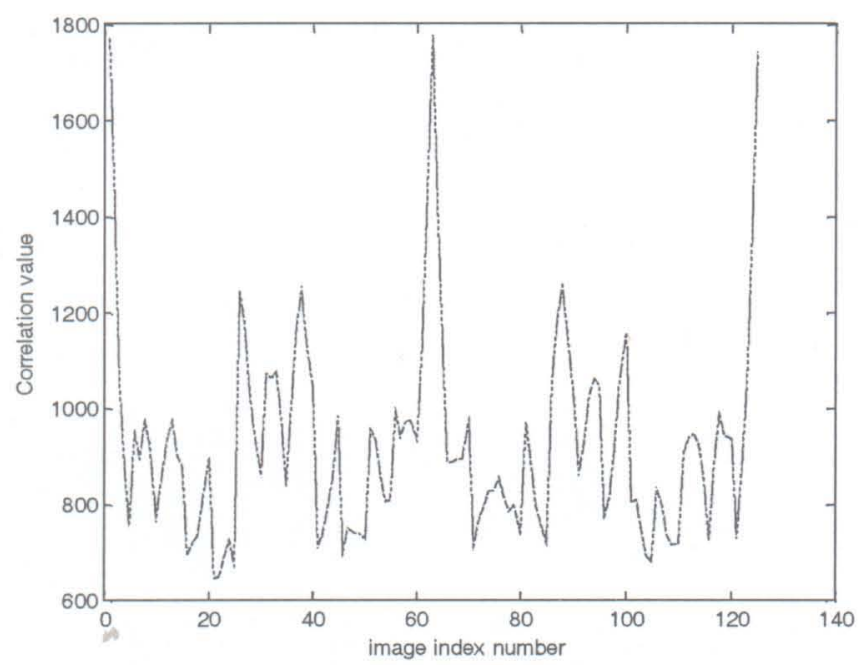

Figure 7: Correlations via ECP BPOSDF

Table 1: Comparison of ECP BPOSDF Correlations

\begin{tabular}{|c|c|c|c|c|c|}
\hline & & & & & $\begin{array}{c}\text { Minimum } \\
\text { From } \\
(0,0,0)-(8,8,8) \\
\text { Range }\end{array}$ \\
\hline $\begin{array}{c}\text { Correlation } \\
\text { Value }\end{array}$ & 69 & 204 & 221 & 1772 & 643 \\
\hline
\end{tabular}

After it was established that a BPOSDF can be designed to perform the first (interclass distinction) step in our two-step pose estimation process, we proceeded to the pose estimation step using BPOFs with a linear estimator. For the BPOF estimator, we chose a construction set of 27 filters. These filters corresponded to the same 27 images used as construction filters for the conjugate matched filter pose estimator. BPOFs were computed for each of the 27 images in the construction set. The $27 \times 27$ correlation matrix was computed, using the BPOFs. Using the $27 \times 27$ correlation matrix as the prescribed correlations, the $3 \times 27$ linear estimator was computed using the same mathematical approach as used for the conjugate filters. The 189 test images were run through the linear estimator, and statistics were compiled. RMS Pitch (out-of-plane) accuracy was 0.75 degrees. RMS yaw (in-plane) accuracy was 0.33 degrees. RMS roll (outof-plane) accuracy was 1.29 degrees. When using the BPOFs, in-plane accuracy is much better than out-of-plane accuracy, and for this particular orientation, our estimator is least sensitive to roll. This makes sense intuitively because 
the BPOFs are highly sensitive to significant changes in the spatial content of the image. At the orientation under study in our class 1, roll motions do not effect large changes in spatial content.

Since the performance of the BPOF-based linear estimator was much worse than the conjugate filter estimator, we proceeded to train an ANN using BPOFs seeking to improve the results. We again used 2-layer backpropagation. The training set comprised the same 125 images used for the conjugate filter ANN. Best results were obtained with 40 hidden neurons and only 14 training epochs. After evaluating 189 test images, pitch accuracy was 0.38 degrees RMS, yaw accuracy was 0.24 degrees RMS, and yaw accuracy was 0.71 degrees RMS. This was a fairly significant improvement over the BPOF linear estimator. To observe the effects of over-fitting, we evaluated statistics on only the 64 test images not in the training set. Results were a pitch accuracy of 0.64 degrees RMS, yaw accuracy of 0.42 degrees RMS, and roll accuracy of 1.23 degrees RMS. The estimates of the non-training poses were about as accurate as the estimates using the linear estimator. This indicates that the BPOF ANN performance is hampered by over-fitting.

\section{Practical Considerations}

In the above sections we have defined a methodology for space vehicle pose estimation and performed a theoretical proof-of-concept. Keeping in mind that this methodology would ultimately be implemented in real hardware, we adapted the methodology to using BPOFs, exclusively real-valued filters, for realization in the spatial light modulators of an optical correlator. In this section we will discuss some observations made during the development and proof of the pose estimation methodology. We will examine the class distinction step and the special considerations that must be made when using BPOSDFs in this phase of the methodology. We then will discuss the quantitative performance of our pose estimator, examining the differences in conjugate filters versus BPOFs and linear estimators versus nonlinear estimators. We will then discuss how our methodology can be extended to full 6DOF pose estimation, including range and in-plane translations. Then we will discuss some of the trades involved in implementation of this pose estimator in an optical correlator versus a digital correlator for space vehicle applications.

For space vehicle pose estimation, if one assumes no a priori knowledge of the coarse pose of the vehicle (that is, the. pose is unbounded), one must first go about determining which subset of poses the vehicle belongs to in order to proceed with the pose estimator described in Figure 1. When one applies the methodology of Figure 1 to a target whose pose is unbounded, two things can happen. First, one would have to design an estimator which yields high accuracy over a very wide range of poses. This would involve a very high-order estimator using a very large set of construction filters. This would also require a very large quantity of correlations to be evaluated in real-time. All of these requirements would be too demanding for the limited resources of a space system. Second, even if one did apply the method of Figure 1 to such an unbounded range of poses, there is a large risk of false detection. That is, the estimator might compute what it thinks is a good pose estimate for an image that does not represent the true pose. Castro encountered this possibility when including a detection flag as one of the states in the pose estimator ${ }^{3}$.

To avoid the difficulties of a complicated high-order estimator and false detection, we proposed the 2-step approach described in Section 2 above. The full 3-axis, 360-degree range of motion is spanned by a finite number of subsets of poses. Each subset would be treated like a class. As section 3 showed (when using conjugate matched filters), a finite set of multilevel SDFs could be designed to span the entire number of classes and identify the class of the test image. When using BPOFs, a finite set of ECP SDFs would work, one BPOSDF for each class. Multilevel and ECP filters were investigated in our research, but that does not preclude the design of other innovative SDFs that could perform interclass discriminations. From our experiences with the BPOFs, for implementation in real hardware, the multilevel SDFs are not very good candidates for this application. A variation of the ECP filters is the preferred design approach, perhaps even a mutual orthogonal function (MOF) SDF for interclass and intraclass distinction ${ }^{5}$ would work in this application.

Table 2 summarizes the quantitative performance of our pose estimator in its various manifestations. All of these results were evaluated with the Space Shuttle located at a range of 50 meters from the camera. By comparison, the specifications for the Advanced Video Guidance System (AVGS), a leading rendezvous and docking sensor, at 30-50 meters are 0.25 degrees in-plane and 0.5 degrees out-of-plane. AVGS specifications from 50-100 meters are 1.2 
degrees in-plane and 2.4 degrees out-of-plane'. At the range of interest, our pose estimator yields theoretical results comparable to the performance of the AVGS. In theory, our pose estimator could do as well as some of the best sensors available. However, our sensor has yet to be studied for sensitivity and robustness to noise sources, both random and colored. It is expected that noise within the correlator, detectors, SLMs and imaging system will degrade the performance reported here.

Table 2: Pose Estimator Performance Results

\begin{tabular}{|l|c|c|c|c|}
\hline $\begin{array}{c}\text { RMS Estimation } \\
\text { Error (degrees) }\end{array}$ & $\begin{array}{c}\text { Linear } \\
\text { Estimator } \\
\text { using } \\
\text { Cojugate } \\
\text { Filters }\end{array}$ & $\begin{array}{c}\text { ANN using } \\
\text { Conjugate } \\
\text { Filters }\end{array}$ & $\begin{array}{c}\text { Lintear } \\
\text { Estimator } \\
\text { using BPoFs: }\end{array}$ & $\begin{array}{c}\text { ANN using } \\
\text { BPOFs }\end{array}$ \\
\hline Pitch (out-of-plane) & 0.22 & 0.22 & 0.25 & 0.38 \\
\hline Yaw (in-plane) & 0.20 & 017 & 0.33 & 0.24 \\
\hline Roll (out-of-plane) & 014 & 0.29 & 129 & 011 \\
\hline
\end{tabular}

Estimators using conjugate matched filters generally work better than BPOFs. There are two reasons for this. First, the cross-correlation amplitude of conjugate filters drops off more slowly (as a function of pose) than it does for BPOFs. This means that the BPOFs are better intra-class discriminators than the conjugate filters, but the BPOFs do not serve well in an estimator which functions, to a large extent, as an interpolator between poses. The second reason is that BPOFs are, in a sense, discrete approximations to the more continuous conjugate filters. The single-bit binarization of a phase-only conjugate filter results in a quantization into only 2 levels whereas the conjugate filter has a continuity of levels. Also, the BPOF is inherently nonlinear. An analogy is a feedback control system designed in the continuous domain which is then discretized for implementation in digital hardware. Additional errors are introduced into control system performance from discretization and its associated phase lag. Analog-to-digital conversion introduces quantization errors and nonlinearities which adversely affect control system performance. So one can intuitively see why BPOFs would yield worse results than conjugate filters in our pose estimator.

One observes from Table 2 that the nonlinear estimators using the artificial neural network generally perform better than the linear estimators do. One would expect this result because the pose/correlation relationship is inherently nonlinear. However, it was pointed out in sections 2 and 3 that the statistics in Table 2 are skewed by the high accuracy of the estimator on the ANN's training images. The performance statistics of the non-training images reveal that the ANN performance on non-training images is not very much better than that of the linear estimator. This finding is a departure from Castro's results on the $2 \mathrm{DOF}$ problem where ANNs dramatically improved estimator performance. Prior to working the 3DOF problem, we investigated the 2DOF problem and reached the same findings as Castro. However, the extension to 3DOF apparently makes the ANN solution more complicated. We believe the 4-dimensional hyperspace (pitch, yaw, roll, correlation) function grows ever more complicated with each added DOF such that the nonlinear function is very difficult to approximate with a finite set of construction filters, even with a neural network. The result was over-fitting between the training poses. We found it very difficult to heuristically overcome the overfitting problem and could not improve the results very much.

For the reasons cited above, we have chosen to implement linear estimators in a hardware demonstration of this pose estimator. Since relatively little performance is to be gained by the neural network, we feel it is not worth the extra effort to generate a multitude of training images and run the algorithms to train the ANNs. Rather, it is more efficient to perform the quick matrix multiplications using a small construction set of images in order to compute the linear estimator. Furthermore, for a space vehicle application, the linear estimator might be the method of choice, after all. A 
linear estimator will take up less memory in an on-board computer than would a high-order ANN. The on-board computation burden will be less with the simple operations inherent to a matrix multiplication. Finally, it will be easier and less controversial to verify and validate a linear estimator than it would be for a neural network. The inherent nonlinearity of the ANN means more resources must be spent on verification and validation in order to cover enough cases to gain confidence in the system's performance. Also, the mere fact of using a neural network makes project managers very nervous about the risks of flying a self-trained system, even though this particular estimator is a static ANN trained and fixed a priori.

We anticipate extending our pose estimation methodology to complete 6DOF state estimation (rotation and translation). We observed that estimating range can be relatively easy with this approach. As the target object moves away from the camera, its size diminishes in the image plane. Consequently, the correlation value decreases as the object size decreases. Though this relationship is not exactly linear, we learned that it is comparable to the pose/correlation relationships for the rotations. Preliminary studies indicated that our pose estimator could independently estimate range (separate from attitude) to an accuracy of about 5 meters over a range from 50-150 meters from the sensor's camera. By comparison, the AVGS specification for range estimation accuracy is 1.67 meters at $50-100$ meters ${ }^{9}$. If one uses the maximum correlation value in the correlation plane as the measurement (as opposed to the correlation value at the correlation plane origin), one could directly identify the in-plane translation of the target vehicle with respect to a camera-fixed coordinate frame. Thus, all 6 degrees-of-freedom can theoretically be estimated from our technique. We anticipate that estimating range along with pitch, yaw and roll could introduce more complexity into both linear and nonlinear estimators. The 4DOF problem now presents a 5-dimensional hyperspace function of correlations. The 4DOF estimator might have a degraded overall accuracy compared to the numbers reported here. This will be investigated further as we proceed to hardware demonstration.

Through the course of developing and verifying our pose estimator, we considered whether it is better to implement this methodology in an optical correlator or a digital correlator. The optical correlator now has the benefit of performing correlations 4000 times per second, potentially yielding high bandwidth. However, the bottleneck limiting the bandwidth might actually reside with the frame rate on the correlation-plane detector. Even though BPOFs. result in degraded estimation performance, BPOFs are only a single bit per pixel, significantly reducing memory volume and data throughput time. On the other hand, digital correlators can use conjugate filters in the estimator, improving overall accuracy. The digital correlator might be lighter weight than the optical correlator because the digital correlator doesn't require a laser and its accompanying power supply. Digital correlators, however, might not yet be as fast as optical correlators despite recent advances in digital signal processing (DSP) technology, and the DSPs might consume more power than the optical correlator's laser. All these factors will be considered in assessing which type of correlator is better suited for space vehicle applications, particularly on microsatellites in which weight, power and volume are a premium.

\section{REFERENCES}

1. T. Ewing, S. Serati, K. Bauchert, "Optical correlator using four kilohertz analog spatial light modulators," Proc. SPIE 5437, pp. 123-133, (2004).

2. J. Outerbridge and D. Gregory, "Optical Correlation Techniques Applied to Spacecraft Docking", Proc. SPIE, Vol. 6245 (paper 6245-15), 2006.

3. A. Castro, Y. Frauel, e. Tepcihin, B. Javidi, "Pose estimation combining synthetic-discriminant-function filters and neural networks." Applied Optics, Vol. 42, No. 29, pp. 5882-5890, (2003)

4. A. Castro, Y. Frauel, e. Tepcihin, B. Javidi, "Pose estimation from a two-dimensional view by use of composite correlation filters and neural networks," Proc. SPIE 4789, pp. 302-309, (2002).

5. D. Casasent, "Unified Synthetic Discriminant Function Computational Formulation," Applied Optics, Vol. 23, No. 10, pp. 1620-1627, (1984).

6. Z. Bahri \& B.V.K. Vijaya Kumar, "Binary Phase-Only Synthetic Discriminant Fucntions (BPOSDFs) Designed Using the Successive Forcing Algorithm." Proc. SPIE 1297 Hybrid Image and Signal Processing II, pp. 188-193, (1990). 
7. J. Campos, A. Marquez, M. J. Yzuel, J. A. Davis, D. M. Cottrell, I. Moreno, "Fully complex synthetic discriminant functions written onto phase-only modulators." Applied Optics, Vol. 39, No. 32, pp. 5965-5970, (2000).

8. J. Taylor, B.A. King III, J. Steincamp, J. Rakoczy, "Genetic Algorithm Phase Retrieval for the Systematic ImageBased Optical Alignment Test Bed." Publications of the Astronomical Society of the Pacific, Vol. 118, pp.-319323, (2006).

9. MC336-0016, Rev. B, "Orbital Express Procurement Specification for the Advanced Video Guidance Sensor (AVGS).” The Boeing Company, Seal Beach, CA, December 13, 2004. 


\section{SDMetrics Entry - Presentations}

Title:

Presenters:

Conference Name:

Location:

Conference Start Date:

Conference End Date:

Date Presented:

Conference Proceedings to Follow:

Organization:
Space Vehicle Pose Estimation via Optical Correlation and Nonlinear Estimation

J. Rakoczy, K. Herren

Defense and Security Symposium

Orlando, FL

$4 / 9 / 2007$

$4 / 13 / 2007$

Yes

VP63 